\title{
El Currículum educacional como campo epistemológico de la Educación: su construcción mediante investigación en el aula
}

The educational Curriculum as an epistemological field of Education: its construction through Research in the classroom

\section{Volumen 21, Número 2 \\ Mayo - Agosto \\ pp. 1-26}

\author{
Relmu Gedda-Muñoz \\ Rafael Guerrero Azócar
}

Citar este documento según modelo APA

Gedda-Muñoz, Relmu., y Guerrero Azócar, Rafael. (2021). El Currículum educacional como campo epistemológico de la Educación: su construcción mediante investigación en el aula. Revista Actualidades Investigativas en Educación, 21(2), 1-26. Doi. 10.15517/aie.v21i2.44683 


\title{
El Currículum educacional como campo epistemológico de la Educación: su construcción mediante investigación en el aula
} The educational Curriculum as an epistemological field of Education: its construction through Research in the classroom

\author{
Relmu Gedda- Muñoz 1 \\ Rafael Guerrero Azócar²
}

\begin{abstract}
Resumen: El presente ensayo se elabora ante la necesidad de problematizar la condición epistemológica de la Educación como una ciencia constitutiva de las ciencias sociales y de la existencia de un campo de conocimiento que le es propio y distintivo. Para ello se propone, para el caso chileno, el currículum educacional como saber propio de la Educación, argumentando que este es el objeto de conocimiento perteneciente a dicha disciplina y por tanto requiere construirse desde sus propias problemáticas situacionales para articular sus dilemas y soluciones. Con el propósito de avanzar y profundizar en la estructura conceptual y metodológica del campo de la Educación, robusteciendo su posición como ciencia, se postula que la investigación en el aula emerge como estrategia para validar la experiencia docente y del estudiantado para otorgar sentido al currículum educacional. En el análisis de las diferentes concepciones del currículum, se decanta por aquella proposición que entiende el currículum como un proceso permanente de construcción de las condiciones propicias para abogar por la emancipación de los sujetos, considerando la idea del tercero incluido y la ecología de los saberes como instancias de validación para su estructuración e implementación. Como conclusión, se establece que un currículum educacional elaborado en base a la evidencia empírica de la propia actividad educativa, es decir, mediante la investigación en el aula, permite considerar a la Educación como ciencia, validando activamente la experiencia del profesorado y estudiantado, en un ambiente expansivo, libre y emancipado, por sobre las disposiciones estrictas de los gobiernos y los currículos estandarizados.
\end{abstract}

Palabras clave: currículum, investigación pedagógica, docente, epistemología.

Abstract: The present essay aims to problematize the epistemological condition of Education as a constitutive branch of social studies as well as existing on a particular and distinctive field of knowledge. In order to achieve this goal it is proposed, for the Chilean case, the educational curriculum as a particular and essential knowledge of education, arguing that is the object of knowledge belonging to that discipline and therefore requires to be built from its own situational problems to articulate its dilemmas and solutions. With the objective of going further and deeper into the conceptual and methodological structure of the field of Education, so as to strengthen its position as a science, it is stated that classroom research emerges as a strategy to validate the teaching and students' experience to justify the importance of the educational curriculum. In the analysis of the different concepts of educational curriculum, it opts for that proposition that understand the curriculum as a permanent process of building the proper conditions to advocate for the subjects' emancipation, considering the idea of the third included and the ecology of knowledge as validating instances for its design and implementation. As a conclusion, it is established that educational curriculum prepared based on empirical evidence of the educational activity itself, that is, through classroom research, allows to consider Education as a science, actively considering the experience of teachers and students, in an expansice, free and emancipated environment, over the strict disposition of governments and standardized curriculum.

Keywords: curriculum, pedagogical research, teachers, epistemology.

\begin{abstract}
${ }_{1}$ Profesional Asesor, Vicerrectoría de Aseguramiento de la Calidad, Universidad Autónoma de Chile, Temuco, Chile. Magíster en Educación con mención en Evaluación Educacional, Universidad de La Frontera, Chile. Doctorando en Sociología, Departamento de Sociología, Universitat Autonoma de Barcelona, España. Dirección electrónica: relmu.gedda@uautonoma.cl ORCID https://orcid.org/0000$\underline{0002-9747-3220}$

2 Profesor de la Facultad de Educación en la Universidad Autónoma de Chile, Temuco, Chile. Magíster en Ciencias Sociales Aplicadas, Universidad de La Frontera, Chile. Dirección electrónica: rafael.guerrero@uautonoma.cl ORCID https://orcid.org/0000-0001-9102-0653
\end{abstract}

Ensayo recibido: 17 de noviembre, 2020

Enviado a corrección: 10 de marzo, 2021

Aprobado: 19 de abril, 2021

Los contenidos de este artículo están bajo una licencia Creative Commons 


\section{Introducción}

El presente ensayo tiene por objetivo problematizar la condición epistemológica de la Educación como una ciencia constitutiva de las ciencias sociales, mediante el currículum educacional como campo de conocimiento distintivo y atingente a ella misma, y que a su vez la provee de una serie de problemáticas, dilemas y soluciones propias. Luego se plantea que, profundizando en la estructura conceptual y metodológica del currículum educacional mediante la investigación en el aula, es posible robustecer la posición científica de la Educación. Por tanto, se analiza y reflexiona en torno a la condición epistemológica de la educación como campo del conocimiento y la pretención de entregarle un carácter científico mediante el currículum educacional y su construcción como objeto de conocimiento mediante la investigación en el aula.

Al respecto, es necesario señalar que en el proceso de desarrollo del campo de la educación se han elaborado distinciones dentro de su espacio de funcionamiento, con el objeto de propiciar intervenciones sistematizadas e intencionadas, dando origen a la diferenciación del fenómeno educativo en diversas sub-áreas, como son el currículum educacional, la planificación, los procesos de actuación pedagógica (que incluyen los contenidos, resultados de aprendizaje, las metodologías de enseñanza, la evaluación, entre otras), así como otros fenómenos altamente relevantes, como las dinámicas de poder al interior del aula entre docente-estudiante, o las diferentes estrategias para que cada institución educativa pueda hacerse cargo de la diversidad de estudiantes que atiende. No obstante, es relevante señalar que en el presente ensayo se abordará el currículum educacional como eje problematizador, en la medida de que se entiende este campo del conocimiento como aquel que pertenece al fenómeno educativo únicamente y que cualquier aspecto referido al currículum se remite, tarde o temprano, a la disciplina de la educación.

Entendiendo el curriculo educacional como saber propio de la Educación, en el proceso de construcción de una epistemología que le otorga una identidad particular y la hace emerger como disciplina, es posible proponer una distinción clara en cuanto a un ámbito del conocimiento humano que se origina en el fenómeno educativo, aún cuando existe una marcada relación de dependencia entre los fenómenos educativos y otras áreas del conocimiento, como la psicología, la sociología y la antropología. Para esto, se profundiza en la importancia de la investigación en el aula que debe aplicarse en Chile, como herramienta definitoria para una correcta implementación, revisión, evaluación y construcción del 
currículum, orientando esta práctica a la validación de la experiencia cotidiana del profesorado en asociación con su alumnado.

Se comienza con una descripción del contexto y definiciones epistemológicas del tema tratado para comprender de mejor manera el rol del currículum educacional en la conformación de un formato que incluya a los actores principales del proceso educativo en la identificación de sus propias necesidades de aprendizaje, para luego señalar las propuestas y fundamentaciones que llevan a indicar al currículum como un saber propio de la Educación, y a la investigación en el aula como elemento que permite el desarrollo de un currículum reflexivo, emancipador y orientado a construir su contexto, reconociendo las prácticas propias de la educación que son relevantes para la disciplina.

\section{Desarrollo del tema}

\subsection{Proposición: Resolver la cuestión de la condición epistemológica de la Educación}

Resulta urgente, en el contexto chileno, pero también latinoamericano y global, reflexionar sobre el carácter científico del campo de la educación desde su epistemología (Zemelman, 2011; Zamudio, 2012), toda vez que el estatus que intenta conquistar y/o mantener frente a las demás ciencias no está del todo legitimado, sobre todo teniendo en consideración que diversos especialistas (Davis, 1987; Touriñan, 2008, Ocampo, 2014) han señalado la debilidad existente en los paradigmas de la educación, pues si bien han sido capaces de construir y establecer terminologías ad-hoc para los fenómenos educativos, éstos responden a situaciones particulares más que a cuestiones sistemáticas y permanentes, obstaculizando la adquisición de un sentido compartido en cuanto a la intervención pedagógica y sus dilemas. Esto implica que las posiciones adoptadas en el campo de la educación podrían limitar el conocimiento hacia la resolución de problemáticas particulares, desgastando la capacidad de la disciplina para avanzar en un sistema conceptual propio de ella misma (Touriñan, 2008).

Es importante señalar que la intelectualidad europea desde el siglo XVII se ha guiado por una razón objetiva, que juega un rol de estructuración ligada a las ciencias exactas, separando de su contexto a cualquier sujeto u organización estudiada. Al respecto, Ortega y Gasset (1986) señala que "bajo la máscara de la objetividad y la verdad, el racionalismo dejó la propia vida humana sin cimientos y sin encaje profundo" (Ortega y Gasset, 1986, p.46), lo que caracteriza a la modernidad como un proyecto que parte desde la premisa del control 
absoluto sobre la vida y las cosas mediante el conocimiento científico doctrinario. En esta forma de abordar la existencia, queda en evidencia que la modernidad está llena de dicotomías y contradicciones, ya que los distintos conceptos tratados están condicionados por el sometimiento y represión de instintos, la supresión de la espontaneidad y el control sobre las diferencias (Castro-Gómez, 2000).

Por otra parte, las ciencias sociales nacieron en esta vorágine eurocéntrica y han sido pieza clave para la legitimación del proyecto moderno y la elaboración de mecanismos de organización y control. Las ciencias sociales poseen y están basadas en un aparato conceptual que está sostenido por un imaginario colonial de carácter ideológico, quedando subordinadas a una función tecnocrática de validación de las experiencias humanas (De Sousa Santos, 2013).

Sin embargo, en la última parte del siglo $\mathrm{XX}$ ha surgido un relato, en reemplazo del metarrelato moderno que legitimaba la hegemonía eurocéntrica, que consiste en la coexistencia de diferentes juegos de lenguaje (Lyotard, 1990), con reglas propias, sin la necesidad de ser evaluado y validado por un ente superior racional y legitimador. En este contexto, en Educación como parte de las ciencias sociales, ya no es prudente hacer una separación entre objeto y sujeto, entre currículum educacional y educandos, entre metodologías de enseñanza y resultados esperados y docentes, sino que "tenemos que dedicarnos a buscar el conocimiento en acción para construir un mundo mejor ... debemos estar inmersos en el objeto de estudio" (Olivera, 2003, p. 28) mediante una preparación adecuada del profesorado, la instalación de una metodología que permita levantar los velos de la diferenciación, cultivando el sentido de pertenencia, un currículum que atienda las especificidades del entorno, la cultura y los territorios en que se implementa y rescatando el valor incalculable de la experiencia docente y del estudiantado. Así, el currículum debe ser pensado y construido colaborativamente por diferentes actores educativos (familia, docentes, estudiantes) "en y desde el acto o acción educativa misma" (Olivera, 2003, p. 54), valorando la interacción social y la negociación de significados para interpretar el mundo, dotando de una característica fundamental al currículum para hacerlo emerger como saber propio de la Educación: el currículum no es un fin o un producto, sino que es un proceso permanente de construcción de los procesos eminentemente educativos desde una perspectiva emancipatoria. 


\subsection{Argumentos para la discusión}

\subsubsection{El currículum educacional y su conceptualización}

Para efectos de comprender de mejor manera el rol del currículum educacional en la conformación de un formato operacional que incluya a los actores del proceso educativo en la identificación de sus propias necesidades de aprendizaje, se debe tener en consideración que existen diversos enfoques y conceptos de currículum, tanto en su contenido como en su profundidad (Sarramona, 1989). Por ejemplo, en conceptualizaciones más clásicas del fenómeno educativo y de cómo se condiciona el currículum educacional, Stenhouse (1998) indica que este se encuentra altamente intervenido por la idea de la planificación por objetivos, propia del paradigma conductista, en el que se expresa un ideario desde la perspectiva del docente y la asignatura como conductas iniciales que se espera que el estudiantado sea capaz de hacer antes que empiece la enseñanza. Esto implica que la educación se convierte en una cuestión de utilizar ciertos medios para lograr determinados fines, lo que conlleva la omisión de elementos que hoy entendemos que son relevantes, pero que queda entredicho su real consideración. Tales elementos son: las especificidades del contexto territorial y sociocultural, la diversidad de estudiantes, la formación inicial docente, los recursos educacionales, el rol de las familias, entre otros.

Las implicancias que tiene esta visión de la planificación por objetivos para con el currículum educacional, es que el currículum se entiende como un componente dentro de un esquema que solo aporta el ordenamiento de los fines y los medios, pues lo primero que se realiza es la especificación de los objetivos de conducta, para luego comprobar el estado inicial de la conducta en el estudiantado, y así recién planificar la implementación del currículum y las actividades de actuación pedagógica, para finalmente comprobar si se han logrado los objetivos trazados (Stenhouse, 1998). Como es posible apreciar, este enfoque entiende la educación como un fenómeno lineal, y por tanto sus elementos constitutivos solo adquieren valor en la medida en que forman parte de una cadena productiva (Gimeno, 1997). Es decir, tanto el currículum educacional como los diferentes elementos intervinientes están predestinados a utilizarse tecnocráticamente, teniendo motivadores extrínsecos para la adhesión a este enfoque, como son la planificación unificada, unívoca y carente de reflexividad pedagógica de los contenidos, el currículum, las estrategias didácticas y la evaluación, pues todos estos elementos están orientados a actuar sobre lo predicho, medir lo predicho y evidenciar resultados sobre lo predicho, lo que deja fuera la incerteza, el resultado del descubrimiento y su explicación epistemológica desde la experiencia docente y del propio 
estudiantado. Este enfoque conductista por objetivos promueve la idea de que el alumnado aprende de forma memorística y reiterativa, donde la noción del aprendizaje es a través del refuerzo (Stassen Berger, 2007) y caracterizándose en que el aprendizaje resulta de una conexión mecánica estímulo-respuesta, donde quien realiza la docencia es quien sabe, enfrentándose a una audiencia que no sabe, de hecho muchas veces sin siquiera considerar al estudiantado como un sujeto realmente, sino que se asume que son objeto del acto educativo (Freire, 2011). El presente ensayo busca dejar en claro que, estando de acuerdo en que existen diversos enfoques que se amparan en diferentes paradigmas para entender el currículum educacional, una parte importante de la docencia y de la planificación educativa se realiza siguiendo los fundamentos conductistas señalados anteriormente, asunto respecto del que existe variada evidencia (García-Huidobro, 2007; Gómez, Santa Cruz y Thomsen, 2007; Carretero, 2009; Viñoles, 2013; Figueroa, Muñoz, Lozano, Zavala, 2017), y es por ello altamente relevante dotar a la educación de un campo de conocimiento propio que le permita problematizar sus conceptos y resolver progresivamente sus dilemas, desde la propia acción y experiencia docente-estudiante.

Así, es posible avanzar hacia conceptos de currículum educacional como el que señala Pansza (2005), quien indica que el currículum representa una serie estructurada de experiencias de aprendizaje que en forma intencional son articuladas con una finalidad concreta: producir los aprendizajes esperados. Sin embargo, esos aprendizajes esperados no pueden ser puramente conceptuales, sino que, en el contexto actual, deben involucrar determinadas competencias, desempeños y actuaciones valóricas o actitudinales (Le Boterf, 2001), de manera que es posible complementar la definición anterior con la de Sanz Cabrera (2003) quien señala que el currículum no solo es un proyecto estructurado respecto a la formación y sus procesos, sino que este se diseña con la finalidad de producir aprendizajes que se traduzcan en formas de pensar, sentir, valorar y actuar, frente a los problemas complejos que plantea la vida social y laboral en un territorio determinado.

Cabe también considerar, en la definición de currículum, que este es un "producto histórico, que se expresa en una propuesta educativa, la cual incorpora de manera tácita o explícita aspectos políticos, ideológicos, epistemológicos, ontológicos y pedagógicos. Está mediado por la realidad política, económica y sociocultural donde se desarrolla" (Rojas, 2012, p. 5), y que para el propósito del presente ensayo es fundamental contemplar todos los elementos involucrados en los procesos de planificación, actuación pedagógica, seguimiento, evaluación educativa y retroalimentación. De esta forma, y si se tiene en cuenta que los 
currículos contemplan diversos elementos contextuales, pedagógicos y operativos, entonces es necesario entenderlo como la organización y disposición de todos los factores comprometidos en el proceso educativo, como son: los recursos y su gestión (espacio y materialidad, tiempo, capacidades docentes y directivas), los aspectos socio-culturales (territorio, composición de los grupos humanos en cuanto a etnia, ruralidad o urbanidad, nivel socioeconómico, entre otros), los elementos pedagógicos (planificación, metodologías de enseñanza, didáctica, evaluación, etc.), y los mecanismos de aseguramiento de calidad que le permiten mejorar con espacios de libertad y flexibilidad. Todos estos factores, adecuadamente coordinados, propician su correcta implementación y aseguran la preparación de las personas para determinados desempeños en un marco previamente definido. Por otra parte, considerar esta definición de currículum educacional permite entenderlo como un proceso en permanente desarrollo, pero que cuenta con momentos e instancias clave para ser puesto en aplicación, y también para ser evaluado y mejorado.

Al respecto, es posible señalar que, para identificar y describir los elementos asociados al currículum educacional, así como a sus posibles puntos de inflexión para convertirse en un saber propio de la educación, este debe entenderse desde su implementación, es decir, desde su puesta en operación. En este sentido, la idea de implementación del currículum se articula en tres grandes áreas o conceptos intervinientes: el contexto socio-cultural, los recursos necesarios para llevar a cabo lo planteado en el currículum y, por supuesto, los elementos pedagógicos. Contemplar el contexto socio-cultural en el que se desarrollará el trabajo a partir de la implementación de un currículum es relevante ya que existe la necesidad de enfocar el trabajo en el aula sobre los elementos que provocan sentido de pertenencia y facilitan el aprendizaje significativo (Ausubel, 1983; Moreira, 2000; 2012; Díaz y Hernández, 2015), sobre todo dados los diferentes territorios en que se implementan los actos pedagógicos y las características diversas del alumnado de un mundo globalizado e intermediado por tecnologías. Los recursos resultan ser un concepto central ya que permite prever los costos, materiales, tiempo y capacidades en términos de profesionales y asistentes de la educación requeridos para avanzar con éxito hacia una correcta implementación del currículum, y por su parte, los elementos pedagógicos constituyen un concepto clave en la implementación del currículum, ya que concentran parte importante de los factores educativos que permitirán el logro de los aprendizajes. Dentro de este último concepto se encuentran los asuntos que, en las definiciones más técnicas antes revisadas, definen el currículum. Es importante señalar que los elementos pedagógicos sientan sus bases en cómo se enseña pedagogía y cómo se 
entiende la formación inicial y el perfeccionamiento docente en un determinado contexto, siendo esto de extrema importancia, toda vez que es el profesorado quien lleva a cabo la actuación pedagógica y quien debe comprender, desde su primera formación profesional, la relevancia y protagonismo que puede tener el currículum que implementa, sobre todo si lo asume como un proceso emancipatorio en permanente construcción, más allá de la sola programación lineal de objetivos y contenidos que se le pudo haber impuesto desde la administración educativa.

Ahora bien, cuando se vinculan los elementos señalados precedentemente, es posible establecer interconexiones entre ellos tres (contexto socio-cultural, recursos y elementos pedagógicos) en la medida en que se caracteriza cada uno:

- El contexto socio-cultural se encuentra mediado por los territorios donde se implementa el currículum y por tanto impacta en la labor del docente, definiendo en gran medida el estudiantado en que se intervendrá, las familias que están detrás de ese grupo de estudiantes, así como el compromiso que puedan manifestar con los procesos educativos de sus integrantes.

- Por otra parte, en cuanto a los elementos pedagógicos, las metodologías de enseñanza se relacionan estrechamente con la didáctica pedagógica, la que debe responder a la diversidad en el grupo de estudiantes, que se relaciona con las diferentes formas de aprender y entender el conocimiento y el mundo, lo cual conlleva una necesaria personalización de la enseñanza atendiendo al contexto socio-cultural donde se inserta el proyecto educativo. También puede considerarse extremadamente importante el que exista una estrecha relación entre las metodologías de evaluación de los aprendizajes y las actividades pedagógicas propuestas, ya que el proceso evaluativo debe desarrollarse sobre aquellos elementos que se contemplaron inicialmente y que se implementaron en el aula, lo que permitirá una correcta verificación de los niveles de logro de los aprendizajes respecto a determinadas competencias, contenidos, habilidades y valores.

- Adicionalmente, al reflexionar en cuanto a la interacción de elementos pertenecientes a los recursos y el contexto socio-cultural, es posible identificar cómo los recursos humanos $y$ los talentos, en cuanto docentes, profesionales asistentes o de profesionales auxiliares a la educación, poseen una serie de expectativas respecto a su estudiantado, que pertenece al primer concepto clave (contexto socio-cultural), y permite que este se vincule a los recursos contemplados en la implementación del currículum. 
El ejercicio reflexivo necesariamente decanta en la aparición de nuevos conceptos para orientar el análisis hacia procesos de evaluación y mejora permanente, como la evaluación del currículum mediante la investigación en el aula (Morán Oviedo, 2004; Porlán Ariza, 2011), el mejoramiento continuo para la innovación curricular (Estebaranz García, 1999; Guzmán, Maureira, Sánchez y Vergara, 2015) y la definición de un Perfil de Egreso (Adam, 2004; Kennedy, Hyland y Ryan, 2006; Möller y Gómez, 2014) para cada nivel educacional como declaración de los propósitos específicos de dichos niveles, idealmente acompañado de un marco de cualificaciones, lo que permitiría desarrollar una evaluación clara y precisa de los logros en la implementación de los currículos, basado en aspectos previamente conocidos y socializados, pudiéndose verificar el estado de avance del alumnado hacia el Perfil declarado, y al mismo tiempo se podría evaluar concretamente el currículum con vistas al mejoramiento continuo y a la innovación curricular adecuada al contexto.

Considerando los planteamientos previamente realizados, se puede identificar que existe una brecha entre las concepciones relativas al currículum educacional y la práctica educativa como tal, lo que conlleva a que se deba avanzar en resolver esta problemática con miras a robustecer la posición de la educación dentro de las ciencias sociales como disciplina con un campo de conocimiento propio.

\subsubsection{Currículum Educacional y la factibilidad de hacerlo emerger como saber propio de la Educación}

En cuanto a la planificación formal del currículum y su intencionalidad relacionada a un rol constitutivo como saber propio de la educación, es importante el aporte al conocimiento curricular de Magendzo (2001), quien destaca que el interés técnico del currículum se dirige a controlar y regular objetos (cosas) y se consigue típicamente mediante la ciencia empíricoanalítica (la perspectiva científico-natural de la investigación científica). Siguiendo en su análisis, reconoce que el interés práctico busca educar el entendimiento humano para informar la acción humana y se consigue por medio de productos que son informes interpretativos y sociales que inducen a actuar de formas determinadas. En especial se utiliza en el sentido de ayudar simplemente a comprender alguna idea o acontecimiento histórico en un contexto interpretativo. Más allá de los intereses técnicos y prácticos, se plantea un tercer tipo de acción: el interés constitutivo del conocimiento emancipador, que es un genuino "interés por la autonomía y la libertad racionales, que emancipen a las personas de las ideas falsas, de las formas de comunicación distorsionadas y de las formas coercitivas de relación social que 
constriñen la acción humana y social" (Magendzo, 2001, p. 71). Esto opera como un contrapunto, tanto a la ciencia social técnica (empírico-analítica) la cual busca la regulación y el control social, así como a la ciencia social práctica (interpretativa), que se orienta a interpretar el mundo. Por su parte, la ciencia social emancipadora revela la manera en que las relaciones de poder distorsionan los procesos sociales amparándose en las lógicas de la dominación y coerción, no solo haciendo inteligibles las relaciones sociales y su lógicas operativas, sino que también buscando construir las condiciones para que las relaciones sociales asimétricas y distorsionadas puedan transformarse, ya sea como acción cooperativa, organizada o lucha política, con el propósito de superar la injusticia en que se fundamentan.

En este sentido, es posible señalar que en Chile el currículum educacional nacional se encuentra definido, en su estructura y en la práctica, como un currículum cerrado, técnico y práctico, que pretende la adquisición, mantención y reproducción de un gran volumen de contenidos, lo cual es propio del paradigma conductista (Rodrigo y Arnay, 1997; Salas, 2002; Gómez et al., 2012). Esto implica que, desconociendo las diferencias naturales y legítimas existentes en el contexto educativo, se busca homogenizar los procesos de aprendizaje, así como la propia labor docente, midiendo la eficacia del sistema en función de pruebas nacionales estandarizadas (Sistema de Medición de la Calidad en Educación -SIMCE- y Prueba de Selección Universitaria -PSU- o la actual Prueba de Admisión Transitoria en Chile), y donde el rol del profesorado queda supeditado a las características de su formación inicial, que les instruye según cómo se enseñaba a mediados del siglo $\mathrm{XX}$, a pesar de que en el discurso generalizado en el ámbito educativo se indique lo contrario (Gómez, Santa Cruz y Thomsen, 2007). A pesar de los esfuerzos realizados en cuanto a la implementación de modelos educativos y pedagógicos actualizados y el desarrollo de una carrera profesional docente, los autores del presente ensayo sostienen que en Chile ha sido muy difícil su aplicación real, debido a los métodos de evaluación centralizados y estandarizados, la formación inicial docente y porque se sigue asumiendo el currículum como un instrumento de programación lineal, de selección de contenidos y de secuencialidad plana y estandarizada para todos los educandos. Este punto es de alta importancia en la formulación de los currículos escolares en Chile, donde la predicción y la cuota de poder de los arquitectos curriculares gubernamentales despojan de autonomía a las unidades educativas, enmarcándolas con sus proyectos educativos obligatorios en ciertas competencias disciplinarias y transversales, que se ajustan más bien al capricho interpretativo de la realidad planificada que a la incertidumbre de un mundo nuevo por venir y por descubrir (Freire, 2005), el cual se vería inmensamente 
enriquecido por la incorporación sistematizada de la experiencia y saberes docentes y las consideraciones del contexto socio-cultural donde se implementan dichos currículos, en base a la investigación en el aula (Morán Oviedo, 2004; Porlán Ariza, 2011).

El ordenamiento del currículum educacional ideal y el material no alcanza dimensiones emancipadoras, y de hecho tiende a homogeneizar desconociendo la diversidad, propiciando un clima que perjudica la inclusión y la contextualización, y donde se asume que el estudiantado aprende del mismo modo y con recetas externas a su interés real. Si se reflexionan estas implicancias en el actual contexto de confinamiento y aislamiento producto de las condiciones sanitarias globales, las diferenciaciones que produce el currículum educacional respecto a las condiciones materiales y no materiales del alumnado podrían resultar aún más complejas que las vividas hasta el momento. De hecho, teniendo claro que, mayoritariamente, éstos aprenden y recopilan experiencias valiosas para su vida tanto fuera del aula como dentro de ella (Hull y Schultz, 2001; Dignath, Büttner y Langfeldt, 2008; Fulton, 2012; Triantafyllou y Timcenko, 2015), una gran debilidad de los enfoques educativos vigentes, sustentados en currículos educacionales de corte conductista (Stenhouse, 1998), radica en que no se aprovecha ni se está de acuerdo con los conocimientos empíricos o las experiencias propias del profesorado y estudiantado en la sala de clases. Ello porque los objetivos predeterminados y cerrados favorecen clases descontextualizadas, y el profesorado es desaprovechado en cuanto a su creatividad y capacidad, considerando además que existen ámbitos del conocimiento en que muchos estudiantes ya han vivido experiencias prácticas y significativas y que a partir de ellas podrían aportar contundentemente a su propio proceso de aprendizaje, así como al de sus compañeros y compañeras. A ello puede adicionarse que, el hecho de que los objetivos por cumplir estén descritos en forma cerrada y sincrónica, produce una distorsión entre los objetivos de la conducta esperada y la naturaleza y estructura del conocimiento y su epistemología, produciendo un distanciamiento entre lo que se hace en las clases y los resultados que se espera que la educación formal produzca, tanto en un nivel cognoscitivo, procedimental y valórico o ético, como desde la perspectiva de la formación ciudadana (Arce Ramírez, 2019).

Desde este punto, se entiende que el currículum educacional no es una mera conjugación de elementos funcionales, sino más bien una práctica sostenida en la reflexión y estrechamente vinculada a sus condiciones de producción y sus prácticas. De allí la importancia de los procesos emancipadores, y no solo de los resultados. En este sentido, resulta ineludible que los docentes sean investigadores sobre su práctica, sus fundamentos y 
consecuencias. Así, es necesario incorporar la idea de que existen diferentes conjuntos de criterios de validez del conocimiento y, por tanto, es posible asumir los conocimientos tradicionales y que provienen de la experiencia como legítimos, toda vez que se les reconoce su condición de práctica epistémica (Olivé et al., 2009), fundamentada en el accionar de grupos humanos y la disposición de determinados medios para alcanzar sus fines, los que se traducen en generación de conocimiento válido para su actuar en un campo social definido.

Sin embargo, como señala Bourdieu (2008), no es posible hablar de epistemología sin considerar la institucionalidad que la genera, y por tanto, es altamente relevante atender a los fundamentos económico-políticos que están a la base de las políticas educativas, de los modelos educativos nacionales y de los proyectos educativos públicos y privados. En este sentido, el sistema educativo chileno se caracteriza por su alto grado de segregación socioeconómica, por sus deficientes resultados en los grupos de menores ingresos (Organización para la Cooperación y el Desarrollo Económico [OCDE], 2009) y por su obstinada orientación a medir logros según resultados en pruebas estandarizadas, todo ello impulsado por un modelo neoliberal que promueve abiertamente la competencia individual e institucional centrada en indicadores que poco o nada nos dicen sobre el aprendizaje real del alumnado. Cabe preguntarse entonces si es que existen alternativas para mover el foco de desarrollo de la educación desde lo estrictamente constatativo y cognoscitivo, hacia elementos que permitan evidenciar competencias, comportamientos, actitudes y desempeños emancipadores que aporten de forma concreta a la convivencia social y al bien común o compartido.

En este sentido resulta relevante comprender que los cambios en las personas, el aula y sus cotidianidades, están supeditadas a los cambios que se produzcan en el centro educativo y, por tanto, los esfuerzos para desarrollar cambios o mejoras deben estar dirigidos a las instituciones (Simons, 1999), a las personas y al propio proceso educativo. Es por esto que la implementación de un currículum no se puede entender si no es articulada con los diferentes actores y entidades involucradas.

\subsubsection{El rol de la Investigación en el aula para contribuir al fortalecimiento del currículum educacional}

Si bien el conocimiento en educación se vincula estrechamente con las actuaciones pedagógicas del profesorado, así como a los procesos de aprendizaje del estudiantado, es necesario reconocer que la acción profesional docente se encuentra supeditada a los modelos 
de formación inicial, así como a las condiciones y recursos presentes en las instituciones educativas, además de las variabilidades de los contextos donde se lleva a cabo. De esta forma, es escasamente posible que cualquier conocimiento en el campo de la educación sea ajeno a su propia praxis, la cual requiere de amplitud, pluralismo y aperturas metodológicas (Touriñan, 2008) para su construcción como objeto de conocimiento. En relación a ello, es importante realizar procesos investigativos en el aula que permitan recabar información para hacer pertinente la educación, sin complejizar en extremo los sistemas de evaluación y seguimiento de la calidad en la educación (Ribeiro, 2007). Hay que priorizar que se tomen en cuenta los elementos fundamentales asociados a las prácticas que definen lo educativo y no aquellos que promueven la homogeneidad, la producción de datos y documentos que carecen de sentido para las instituciones y las personas.

Se debe comenzar a tratar sobre las particularidades de cada región, territorio, zona urbana o rural, etc., haciendo uso de la experiencia en el aula, tomando, eso sí, un cuerpo de elementos mínimos comunes a modo de currículum nacional de base, que permita la coexistencia de un marco unificado de conocimientos, actitudes, destrezas y desempeños (orientándose al desarrollo de competencias) mínimos exigibles, y un gran espectro de elementos propios del contexto que se enseñen porque tienen sentido para las personas que los viven.

Si bien un cambio al respecto requiere un proceso adaptativo, no solo paradigmático sino que simbólico y a nivel de políticas públicas, es posible pensar en determinadas alternativas que permitan mejorar progresivamente la realidad educativa del país hoy, con miras a mejorar tanto los indicadores de rendimiento, como nuestra capacidad como país para educar personas más capaces y más interesadas en la convivencia y el progreso social que en sus propios desempeños individuales. Es necesario reconocer que tenemos un problema y que existen alternativas para su solución. Según la perspectiva de los autores, para esto es fundamental dotar a las carreras de pedagogía de contenidos precisos y efectivos para realizar investigación en el aula (Morán Oviedo, 2004; Porlán Ariza, 2011). Este elemento resulta fundamental ya que la única forma real de evaluar el impacto en la implementación de un currículum nacional reside en los hallazgos concretos que ocurren en su aplicación a nivel de las aulas de clases. Este tipo de capacidades instaladas en el personal docente y de apoyo a las labores académicas, además de las prácticas sistematizadas de divulgación de hallazgos y buenas prácticas docentes, permitiría retroalimentar y fortalecer el currículum generando un círculo virtuoso de mejoramiento continuo, innovación curricular y perfeccionamiento de 
estrategias pedagógicas centradas en el contexto, facilitando que cada región, provincia o inclusive comuna implemente sus propias iniciativas microcurriculares (Sistema de Créditos Transferibles de Chile [SCT-Chile], 2015). En este sentido, y desde una perspectiva operativa, la investigación en el aula llevada a cabo de manera periódica y permanente, permite que cada establecimiento pueda relevar hallazgos significativos tanto en la práctica educativa como en la implementación curricular, construyendo progresivamente un cuerpo de conocimientos y competencias que resultan relevantes para su contexto, y que a su vez permiten mejorar el diseño e implementación de los curriculos educacionales de manera consecutiva en cada período académico.

Una vez constituido el currículum educacional como saber propio de la educación, y la investigación en el aula como su motor de dilemas y profundizador de soluciones, se hace posible pensar en tensionar el imaginario colectivo docente, el que cuenta con determinadas preconcepciones mentales para la comprensión de su mundo y los saberes que son inteligibles en educación (Nicolescu, 1996). La realidad demuestra que la distancia entre las disciplinas agudiza y hace cada vez más urgente la comprensión integrada del mundo para encontrar respuesta a las necesidades humanas. En este sentido, la transdisciplinariedad (Nicolescu, 1996), que es lo que se construye entre las disciplinas, a través de las disciplinas y más allá de las disciplinas, otorga una vía de acción. Su propósito radica en la comprehensión del mundo presente, como una dinámica originada por la acción de diversos niveles de realidad simultáneos, lo que sería impensado para la perspectiva clásica, que entiende el mundo desde la compartimentación de los objetos de conocimiento posible mediante las diferentes disciplinas. Siendo así, la transdisciplinariedad puede manifestarse por medio de la acción investigativa del profesorado, cuando se integran diferentes supuestos, conceptos 0 metodologías para enfrentar una problemática educativa concreta. No obstante, es importante no caer en lo que, diferentes autores, denominan el tercero excluido en investigación (Caillé, 1992; Salazar y Soriano, 2011; Brito, 2019), donde la relación entre sujeto y objeto se encuentra fracturada, mediada por las herramientas inventadas por el propio sujeto investigador. Esta condición cambia en la visión transdisciplinaria, incorporando la noción de tercero incluido (Nicolescu,1996). Esta inclusión sería la forma en que el sujeto se incorpora en el objeto que estudia, en particular en las ciencias sociales y en la educación, en que la realidad vigente e inclusiva del propio sujeto -el profesorado- en su trabajo diario en el aula, haciéndolo protagonista y no solo observador neutral del aula con sus estudiantes. La dinámica del conocimiento disciplinario desde la transdisciplinariedad permite, entonces, aproximarse a 
la realidad social, cultural y educativa abriendo una relación integrada entre profesorado y estudiantado, y entre fenómeno educativo y su resolución en el aula.

En términos concretos, es altamente pertinente, para realizar esta labor, la Teoría Fundamentada (TF) (Strauss y Corbin, 2002; Charmaz, 2013), toda vez que propone los métodos que permiten reconocer cada elemento presente en un espacio social dotado de sentido para los sujetos, evitando separar los componentes que en última instancia permiten reconocer esa realidad. La investigación en el aula podría ser así, un factor de enorme valía al respecto, ya que daría especial importancia al reconocimiento de las prácticas de construcción del saber en la relación docente-estudiante-institucion educativa-familia-entorno. La idea de que el conocimiento se construye acudiendo a la realidad concreta y no a priori, que inclusive el objeto de estudio se elabora empapándose de la experiencia, considerando que la investigación en sí se elabora y reconstruye constantemente durante el acceso del investigador al entorno estudiado, son principios básicos de la TF, dándole un carácter abductivo y retroductivo, evidenciando siempre los fundamentos de los hallazgos, entregándoles a éstos el valor y rigor de lo científico. En este mismo sentido, una premisa esencial para la TF es que quienes realicen el trabajo investigativo deben asegurar que se efectúa una constante vigilancia epistemológica (Bourdieu, Chamboredon y Passeron, 2004), que permita realizar procesos de acercamiento profundo a la realidad dejando entre paréntesis las prenociones de los investigadores, y provocando las rupturas adecuadas con las nociones comunes, lo que no significa desconocer o evitar ser crítico del contexto o las condiciones económico-políticas que existen, sino tener absoluta claridad de su influencia sobre el fenómeno del aula observado y sobre el investigador como sujeto social, propiciando procesos emancipadores de generación de conocimiento.

Para ello, se debe cumplir el supuesto de que la comunidad educativa (docentes, estudiantes, familia y establecimiento educacional), manifiestan libre y abiertamente la intencionalidad y compromiso de mejorar la calidad educativa mediante la contextualización del currículum educativo, asegurando su pertinencia mediante la investigación en el aula. Una vez existe acuerdo en ello, el profesorado adquiere, legítimamente, la condición de investigador activo en el aula, pudiendo iniciar procesos reflexivos y emancipadores sobre la práctica educativa, ya sea mediante proyectos de mediano y largo aliento, por ejemplo sobre los resultados obtenidos en un curso, en un grupo de asignaturas afines o sobre la integración estudiantil a lo largo de un período de tiempo determinado. También puede desarrollarse investigación en el aula mediante instancias investigativas puntuales para mejorar el proceso 
de enseanza durante un curso académico, construyendo objetos de estudio acotados y definidos de común acuerdo con el estudiantado o sus familias, transformando prácticas educativas tradicionales, a saber, expositivas, verticales y unidireccionales, en actuaciones pedagógicas dotadas de sentido para el estudiantado en su contexto, develando, en el proceso, las diferentes formas en que la definición estandarizada y centralizada de los currículos opera para enseñar ciertos contenidos y desempeños por sobre otros. Lo importante es que, si el proceso o los resultados decantan en mejores logros educativos o en aprendizajes significativos para la comunidad educativa, entonces el profesorado podrá reflexionar sobre las implicancias y efectos que conlleva su actuación pedagógica, tanto la tradicional como la innovada, y así evaluar la idoneidad de reproducir las estrategias desarrolladas, ya sea en otros cursos o en períodos académicos suscesivos. En estos casos, la investigación en el aula se encuentra constituida por la actividad reflexiva del docente, quien registra y analiza el proceso de elección de contenidos y de construcción de resultados de aprendizaje mediados por el consentimiento del estudiantado o la comunidad educativa, a la vez que registra y analiza los resultados de su actuación pedagógica, junto con los efectos que se han logrado en los y las estudiantes. Dicho proceso puede sistematizarse de manera sencilla en base instrumentos ad-hoc que contemplen ciertos campos para redactar las reflexiones del docente, como un campo para los objetivos de intervención, los acuerdos tomados por la comunidad educativa, las estrategias pedagógicas implementadas (incluyendo los mecanismos evaluativos utilizados), y luego reflexiones y retroalimentación respecto a los resultados obtenidos, con foco en mejorar la estrategia para una siguiente aplicación, y progresivamente su consideración para la construcción de un currículum educativo contextualizado.

Hablar de investigación en el aula no significa formar metodólogas y metodólogos, sino la construcción e instalación de capacidades para el ordenamiento, sistematización y análisis riguroso de hallazgos en el aula, centrándose esencialmente en aquello que para docentes y estudiantes es relevante en términos del propósito de un proceso educativo. Así, es de primera necesidad relevar la trascendencia de la experiencia y los saberes propios de los profesores en el contexto de su actuación concreta, reflexionando y analizando su quehacer y fundamentos con el objeto de mejorar sus prácticas. En este sentido, De Sousa Santos (2003), hace un profundo análisis de cómo se desperdicia la experiencia en general de todo aquello que ocurre en la cotidianidad. Esto acontece por una excesiva valoración de la ciencia entregándole el poder de decidir entre lo verdadero y lo falso, generando un amplio desconocimiento sobre la experiencia por no ser mensurable, y también porque esa ciencia se 
sostiene sobre las lógicas de pensamiento de la sociedad occidental, que delimita la realidad mediante dicotomías (De Sousa Santos, 2013). Así, sería pertinente atender a la ecología de los saberes (De Sousa Santos, 2013), en el sentido de que es indispensable revalorizar los conocimientos que emergen de la intervención concreta en los contextos, en este caso relacionado a la propia generación de conocimientos que se producen en el aula, lo cual involucra tanto al profesorado, como al estudiantado, las familias, las instituciones educativas y la propia comunidad, igualando su categoría de saber epistémico al de la ciencia racionaltecnológica. Esto permitiría la contextualización curricular, legitimando los diferentes tipos de conocimiento (Toruño, 2020) que emergen del actuar docente y pedagógico.

De esta forma, se podría plantear aquello que señala González Velasco (2009), cuando indica que el docente no es solo un profesional que se desempeña dentro del aula, sino que es una persona capaz de enseñar y a la vez aprender en todo espacio y tiempo. Esto se debe a que es un aprendiz permanente y entiende que el proceso de enseñanza-aprendizaje y la didáctica asociada es un sistema complejo constituido por diversos factores humanos (desde lo biológico hasta los recursos materiales, pasando por aspectos psicológicos, emocionales y culturales), además de que entiende que el aula no es solo una instalación, un recinto, sino que se transforma en un espacio dentro de la conciencia de quién aprende, a la vez que de quién enseña.

En un mundo globalizado e intermediado por tecnologías, ni profesores ni estudiantes le encontrarán sentido al experimento cotidiano de la clase mientras lo que se enseñe no responda a aquellos elementos que, en contexto, le otorgan valor a sus propias experiencias. El genuino interés por el otro, tanto de parte del profesorado como por parte del estudiantado, las familias y las instituciones educativas, es aquello que puede constituir la piedra angular sobre la que se base la investigación en el aula para cumplir este propósito de validar la experiencia cotidiana en educación, y así construir progresivamente currículos que tengan verdadero sentido para la vida de las personas.

\section{Conclusiones}

El ensayo presentado cuestiona la condición epistemológica de la educación como una ciencia, y al mismo tiempo propone el currículum educacional como el campo de conocimiento propio de la educación, no siendo esta pretención arbitraria, sino fundamentada en la búsqueda de aquel elemento constitutivo de la disciplina de la educación que resulte únicamente abordable desde la educación en sí misma. La educación en su concepto más 
amplio debiera concebirse como un proceso dinámico entre las personas, con metas y contribuciones para que los sujetos protagonicen su actuación emergiendo desde un espacio protegido, seguro y emancipado, para que asuman en conciencia su rol de perfeccionarse permanentemente. Emanciparse para concretar sus utopías o anhelos, coparticipar de los cambios de su entorno y sentirse inmerso en una sociedad que avanza junto a ellos, responsabilizándose como un sujeto activo de la realidad cambiante en que vive.

El currículum educacional en Chile se ha entendido como una herramienta con propósitos orientadores para conformar una estrategia nacional unificada de selección de contenidos culturales que se despliegan en los sistemas escolares, por tanto transita contemplativamente entre el profesorado, habiendo considerado los mandatos estatales que instruyen a directivos y docentes a seguir pautas centralizadas de acción pedagógica, disminuyendo lesivamente su creatividad, su reflexión y su sentido de libertad. Teniendo estas consideraciones, se propone comprender y robustecer la posición del currículum educacional para adecuar las formas de implementar las actuaciones pedagógicas, haciéndolas más pertinentes a los territorios diferenciados donde se evidencian estos desempeños, así como a los diversos intereses de los aprendices en el marco del nuevo enfoque pedagógico centrado en el estudiantado y el significado que estos le atribuyen a lo que aprenden.

Se ha propuesto situar la tarea del profesorado en los centros educativos como una excursión investigativa y emancipadora en el aula, que les permita construir información relevante y espacios de libertad estratégica de cara a las realidades concretas vivenciadas por el estudiantado y la voz de sus padres y apoderados que demandan progresivamente mayor calidad educativa que la que se tiene hoy en los colegios en Chile. La amplia literatura al respecto, sumado a las evidencias empíricas, demuestran que el currículum ideal del Estado se distancia críticamente del currículum material concreto que utiliza el docente de aula. Prima desde el aparato gubernamental la disposición a incentivar un currículum homogéneo para dar sentido a una mal entendida unidad de contenidos culturales a contrapelo del nuevo discurso social, centrado en atender las diferencias de todo tipo a partir de la educación (Banks, 2012; Schuelka, Johnstone, Thomas y Artiles, 2019).

En la medida que se reconocen los saberes, la experiencia y la práctica en el análisis y pensamiento de la realidad como forma de conocimiento científico mediante la investigación en el aula, existe una gran posibilidad de generar un salto cualitativo en la forma en que se estudia y llevan a cabo los trabajos en el currículum a diferentes niveles (estratégico, planificación, dirección, ejecución, evaluación y retroalimentación), pues todos ellos se verían 
potenciados por el reconocimiento del valor de la experiencia en la realidad desde una perspectiva emancipadora. Si se normaliza la utilización de los saberes para fundamentar y comprender los fenómenos educativos, entonces se estará más cerca de asegurar que la forma en que aprende nuestra sociedad puede mejorarse día a día. Es así que el currículum educacional emancipador se constituiría en saber propio de la educación, siendo el principal soporte de las problematizaciones en la disciplina, y al mismo tiempo la investigación en el aula emergería como una oportunidad invaluable para relevar la importancia del currículum, junto con sus atributos y potencialidades, permitiendo además pasar de un currículum centrado en la instrucción a otro centrado en el aprendizaje, ya que la validación de la experiencia obliga a tener en perspectiva nuevas formas de enseñar, evaluar y por supuesto, aprender.

De esta forma, la investigación en el aula, llevada a cabo con los métodos adecuados, emerge como una alternativa altamente plausible para contribuir a los procesos de diseño, planificación e implementación curricular, permitiendo posicionar a la Educación como disciplina con un campo de saberes propios, levantando y validando conocimientos desde la práctica y experiencia cotidiana, y desarrollando con el tiempo métodos y teorías propias que le sustenten, lo que en última instancia permitirían robustecer la posición epistemológica de la propia disciplina de la Educación.

El cambio de enfoque investigativo, donde la tradición académica del tercero excluido dé paso a un tercero incluido, constituido en el docente comprometido con lo que descubre, permitirá al profesorado sentirse parte del proceso de construcción de un currículum educacional pertinente a las necesidades del entorno, sensible a los cambios del contexto vivencial de sus estudiantes.

Los alcances de las reflexiones desarrolladas en este ensayo, se encuentran estrechamente relacionados a la capacidad y voluntad de los gobiernos y las instituciones educativas formadoras para preparar al profesorado en Chile para dar un salto de espectador a protagonista respecto a la construcción de los currículos educativos, situación del todo compleja dada la concentración de poder en el Estado, el cual contiene y restringe todo atisbo de innovación que suponga una pérdida del control sobre lo que se enseña. No obstante, en el presente ensayo se indica que no se presenta contradicción ninguna en un currículum educacional nacional y una adaptacion local, pues el profesorado sabe, por su formación académica, que contribuye a la formación de sujetos para la sociedad que está enmarcada de deberes y obligaciones que deben ser enseñados en las escuelas. El cómo, cuándo y con qué se enseña, es tarea de los profesionales de la educación, no con docentes pasivos e 
inmovilizados por las instrucciones gubernamentales, sino un explorador de saberes de la comundad de la que es parte y desde allí, generando estrategias para reinventar la planificación, diseño e implementación de currículos educacionales, movilizar los saberes, reconstruyendo espacios de libertad académica. Ello fortalecería la perpectiva de un sujeto social único pero a la vez autónomo y respetuoso de las diferencias, y que a su vez permite conciliar la necesidad de robustecer la posición epistémica de la educación, la disposición y profundización de sus planteamientos conceptuales y metodológicos, y asistir en la concreción de su fin último, como es permitir la co-construcción de ideas, identidades y personas orientadas al bien común, más humanistas y socialmente comprometidos con su quehacer y contribución a la sociedad.

\section{Referencias}

Adam, Stephen. (2004). Using learning outcomes. Recuperado de http://aic.lv/bolona/Bologna/Bol semin/Edinburgh/S Adam Bacgrerep presentation.pdf

Arce Ramírez, Henry. (2019). Competencias ciudadanas: una reconstrucción conceptual en el marco de la Educación Cívica costarricense. Revista Actualidades Investigativas en Educación, 19(2), 1-20. doi: https://doi.org/10.15517/aie.v19i2.36904

Ausubel, David. (1983). Teoría del aprendizaje significativo. Fascículos de CEIF, 1(1-10). Recuperado de http://files.gersain.webnode.es/2000000267721a781a1/Aprendizaje significativo.pdf

Banks, James. (Ed.). (2012). Encyclopedia of diversity in education. New York, Estados Unidos: Sage Publications.

Bourdieu, Pierre. (2008). Homo Academicus. Buenos Aires, Argentina: Siglo XXI Editores.

Bourdieu, Pierre., Chamboredon, Jean-Claude., y Passeron, Jean-Claude. (2004). El oficio de sociólogo. Buenos Aires, Argentina: Siglo XXI Editores.

Brito, Carlo Fabián. (2019). El tercero excluido en el contexto áulico y su impacto en el aprendizaje. Revista Vinculando. Recuperado de http://vinculando.org/educacion/eltercero-excluido-en-el-contexto-aulico-y-su-impacto-en-el-aprendizaje.html

Caillé, Philippe. (1992). Uno más uno son tres: la pareja revelada a sí misma. Barcelona, España: Paidós.

Carretero, Mario. (2009). Constructivismo y Educación. Buenos Aires, Argentina: Editorial Paidós. 
Castro-Gómez, Santiago. (2000). Ciencias sociales, violencia epistémica y el problema de la invención del otro. En La colonialidad del saber: eurocentrismo y ciencias sociales. Perspectivas latinoamericanas (pp.145-161). Buenos Aires, Argentina: CLACSO. Recuperado de http://biblioteca.clacso.edu.ar/clacso/sursur/20100708045330/8 castro.pdf

Charmaz, Kathy. (2013). La teoría fundamentada en el siglo XXI: Aplicaciones para promover estudios sobre la justicia social. . En: N. K. Denzin y Y. S. Lincoln (Comps.), Estrategias de investigación cualitativa (pp. 270-325). Buenos Aires, Argentina: Editorial Gedisa.

Davis, Wayne. (1987). Educational research in the professions: Paradigms, peer review and promise. Professions Education Research Notes, 9(1), 4-9.

Díaz, Frida., y Hernández, Gerardo. (2015). Constructivismo y aprendizaje significativo. En Estrategias docentes para un aprendizaje significativo (pp.13-33). México: Mc Graw Hill. Recuperado http://metabase.uaem.mx/bitstream/handle/123456789/647/Constructivismo.pdf?seque $\underline{\text { nce }=1 \text { \&isAllowed }=y}$

Dignath, Charlotte., Büttner, Gerhard., y Langfeldt, Hans-Peter. (2008). How can primary school students learn self-regulated learning strategies most effectively? A meta-analysis on self-regulation training programmes. Educational Research Review, 3(2), 101-129. Recuperado de https://eric.ed.gov/?id=EJ813065

Estebaranz García, Araceli. (1999). Didáctica e innovación curricular. Sevilla, España: Editorial Universidad de Sevilla.

Figueroa, Helen., Muñoz, Karlita., Lozano, Edwin., y Zavala, Diego. (2017). Análisis crítico del conductismo y constructivismo, como teorías de aprendizaje en educación. Revista Órbita Pedagógica, 4(1), 1-12. Recuperado de http://refcale.uleam.edu.ec/index.php/enrevista/article/view/2312

Freire, Paulo. (2005). Pedagogía del Oprimido. Distrito Federal, México: Siglo XXI.

Freire, Paulo. (2011). La educación como práctica de libertad. Distrito Federal, México: Siglo $\mathrm{XXI}$.

Fulton, Kathleen. (2012). Upside down and inside out: Flip your classroom to improve student learning. Learning \& Leading with Technology, 39(8), 12-17. Recuperado de https://eric.ed.gov/?id=EJ982840

García-Huidobro, Juan. (2007). Desigualdad educativa y segmentación del sistema escolar. Consideraciones a partir del caso chileno. Pensamiento Educativo: Revista de Investigación Educacional Latinoamérica, 40(1), 65-85. Recuperado de http://ojs.uc.cl/index.php/pel/article/view/25489

Gimeno, José. (1997). La pedagogía por objetivos: Obsesión por la eficiencia. Madrid, España: Morata. 
Gómez, Viviana., Guerra, Paula., Santa Cruz, Josefina., Thomsen, Paulina., Rodríguez, Constanza., y Beas, Josefina. (2012). Collaborative reflections dyads: building new meanings onto learning and teaching. Electronic Journal of Research in Educational Psychology, 10(2), 271-310.

Gómez, Viviana., Santa Cruz, Josefina., y Thomsen, Paulina. (2007). En busca del cambio conceptual del profesor en el contexto de la formación permanente. REICE. Revista Iberoamericana sobre Calidad, Eficacia y Cambio en Educación, 5(5), 27-31. Recuperado de https://www.redalyc.org/pdf/551/55121025004.pdf

González Velasco, Juan Miguel. (2009). Didáctica Crítica desde la transdisciplinariedad, la complejidad y la investigación: De cara a los retos y perspectivas educativas del devenir de nuestros tiempos. Revista Integra Educativa, 2(1), 63-74. Recuperado de http://www.scielo.org.bo/scielo.php?pid=S1997$\underline{40432009000100004 \& \text { script=sci abstract }}$

Guzmán Droguett, María., Maureira Cabrera, Óscar., Sánchez Guzmán, Alejandra., y Vergara González, Adriana. (2015). Innovación curricular en la educación superior: ¿Cómo se gestionan las políticas de innovación en los (re) diseños de las carreras de pregrado en Chile?. Perfiles educativos, 37(149), 60-73. Recuperado de http://www.scielo.org.mx/pdf/peredu/v37n149/v37n149a4.pdf

Hull, Glynda., y Schultz, Katherine. (2001). Literacy and learning out of school: A review of theory and research. Review of educational research, 71(4), 575-611. doi: https://doi.org/10.3102\%2F00346543071004575

Kennedy, Declan., Hyland, Áine., y Ryan, Norma. (2006). Writing and using learning outcomes: a practical guide. Cork, Irlanda: University College Cork.

Le Boterf, Guy. (2001). Ingeniería de las competencias. Barcelona, España: Ediciones Gestión.

Lyotard, Jean-François. (1990). La condición postmoderna. Informe sobre el saber. México: Rei.

Magendzo, Abraham. (2001). La educación en derechos humanos: Diseño problematizador. Revista Latinoamericana de Derechos Humanos-DEHUIDELA, 7(1), 65-74. Recuperado de https://www.corteidh.or.cr/tablas/r24460.pdf

Möller, Isabel., y Gómez, Héctor. (2014). Coherencia entre perfiles de egreso e instrumentos de evaluación en carreras de educación básica en Chile. Calidad en la educación, (41), 17-49. doi: http://dx.doi.org/10.4067/S0718-45652014000200002

Morán Oviedo, Porfirio. (2004). La docencia como recreación y construcción del conocimiento Sentido pedagógico de la investigación en el aula. Perfiles educativos, 26(105-106), 41 72. Recuperado de http://www.scielo.org.mx/scielo.php?pid=S0185$\underline{26982004000100003 \& \text { script }=\text { sci abstract\&tlng=es }}$

Moreira, Marco Antonio. (2000). Aprendizaje significativo: teoría y práctica. Madrid, España: Visor. 
Moreira, Marco Antonio. (2012). ¿Al final, qué es aprendizaje significativo?. Revista Qurriculum, (25), 29-56. Recuperado de https://riull.ull.es/xmlui/handle/915/10652

Nicolescu, Basarab. (1996). La Transdisciplinariedad. Manifiesto. México: 7 Saberes.

Ocampo, Aldo. (2014). Consideraciones epistemológicas para una educación inclusiva. Investigación y Postgrado, 29(2), 83-111. Recuperado de https://www.redalyc.org/articulo.oa?id=65848281005

Olivé, León., De Sousa Santos, Boaventura., Salazar, Cecilia., Antezana, Luis., Navia, Wálter., Valencia, Guadalupe ... Tapia, Luis. (2009). Pluralismo epistemológico. La Paz, Bolivia: Editorial Muela del Diablo; CLACSO; CIDES; UMSA.

Olivera, Eduardo. (2003). Reflexiones en torno a la reforma educacional chilena. Revista electrónica Diálogos Educativos. 3(5), 27-61. Recuperado de https://dialnet.unirioja.es/descarga/articulo/2095601.pdf

Organización para la Cooperación y el Desarrollo Económico (OCDE). (2009). Revisión de Políticas Nacionales de Educación: La Educación Superior en Chile. París, Francia: Organización para la Cooperación y el Desarrollo Económico; Banco Mundial. Recuperado de https://ciperchile.cl/wp-content/uploads/LINK6.pdf

Ortega y Gasset, José. (1984). La historia como sistema. En Historia como sistema y otros ensayos filosóficos (pp-29-95). Madrid, España: Sarpe

Pansza, Margarita. (2005). Elaboración de programas. En Operatividad de la didáctica (2ª ed.) (pp. 9-42). México: Gernika,.

Porlán Ariza, Rafael. (2011). El maestro como investigador en el aula: investigar para conocer, conocer para enseñar. En: O. Morán (Comp.), Docencia e investigación en el aula: Una relación imprescindible (pp.25-45). México: Ediciones IISUE-UNAM.

Ribeiro, Janine. (2007). Evaluación de posgrado: experiencia y desafíos en Brasil. En Educación Superior y Sociedad: universidades latinoamericanas como centros de investigación y creación de conocimiento (pp. 223-236). Caracas, Venezuela: IESALCUNESCO.

Rodrigo, María José., y Arnay, José. (1997). La construcción del conocimiento escolar. Barcelona, España: Paidós.

Rojas, Carolina. (2012). Participación de los y las docentes en la transformación curricular, Revista Actualidades Investigativas en Educación, 12(1), 1-18. doi: https://doi.org/10.15517/aie.v12i1.10263

Salas, Flora. (2002). Epistemología, educación y tecnología educativa. Revista Educación, 26(1), 9-18. Recuperado de https://www.redalyc.org/pdf/440/Resumenes/Resumen 44026102 1.pdf 
Salazar, Omar., y Soriano, Jairo. (2011). Las leyes de tercero excluido y contradicción como valores límite en lógica difusa. Ingeniería, 16(1), 50-59. Recuperado de https://www.redalyc.org/pdf/4988/498850172005.pdf

Sanz Cabrera, Teresa. (2003). El currículum. Su conceptualización. En: M. González, A. Hernández, H. Hernández Fernández y T. Sanz Cabrera, Currículo y formación profesional. La Habana, Cuba: Departamento de Ediciones e Imprenta Instituto Superior Politécnico José Antonio Echeverría.

Sarramona, Jaime. (1989). Fundamentos de educación. Barcelona, España: Grupo Editorial CEAC.

Schuelka, Matthew., Johnstone, Christopher., Thomas, Gary., y Artiles, Alfredo (Eds.). (2019). The SAGE handbook of inclusion and diversity in education. United Kingdom: SAGE.

Sistema de Créditos Transferibles de Chile (SCT-Chile). (2015). Manual para la implementación de Sistema de Créditos Académicos Transferibles SCT-Chile (3ª ed.). Santiago, Chile: Consejo de Rectores de las Universidades Chilenas.

Simons, Helen. (1999). Evaluación democrática de instituciones escolares. Madrid, España: Ediciones Morata.

De Sousa Santos, Boaventura. (2003). Crítica de la razón indolente: Contra el desperdicio de la experiencia. Para un nuevo sentido común: la ciencia, el derecho y la política en la transición paradigmática. Bilbao, España: Editorial Desclée de Crouwer.

De Sousa Santos, Boaventura. (2013). Descolonizar el saber, reinventar el poder. Santiago, Chile: Editorial LOM.

Stassen Berger, Kathleen. (2007). Psicología del Desarrollo: Infancia y adolescencia. Madrid, España: Editorial Médica Panamericana.

Stenhouse, Lawrence. (1998). La investigación como base de la enseñanza. Madrid, España. Ediciones Morata.

Strauss, Anselm., y Corbin, Juliet. (2002). Bases de la investigación cualitativa. Técnicas y procedimientos para desarrollar la teoría fundamentada. Antioquia, Colombia: Editorial Universidad de Antioquía, Colombia.

Toruño Arguedas, César. (2020). Marcos conceptuales para un currículo crítico: una propuesta desde la pedagogía crítica brasileña, española y estadounidense. Revista Actualidades Investigativas en Educación, 20(1), 1-27. doi: https://doi.org/10.15517/aie.v20i1.40124

Touriñan, José Manuel. (2008). Teoría de la educación: investigación disciplinar y retos epistemológicos. Magis. Revista Internacional de Investigación en Educación, 1(1), 175194. Recuperado de https://www.redalyc.org/pdf/2810/281021687012.pdf 
Triantafyllou, Evangelia., y Timcenko, Olga. (2015). Out of classroom instruction in the flipped classroom: The tough task of engaging the students. Presented at International Conference on Learning and Collaboration Technologies (pp. 714-723). Recuperado de https://www.researchgate.net/publication/277016408 Out of Classroom Instruction in the Flipped Classroom The Tough Task of Engaging the Students

Viñoles, Máximo. (2013). Conductismo y constructivismo: modelos pedagógicos con argumentos en la educación comparada. HumanArtes. Revista Electrónica de Ciencias Sociales y Educación, 2(3), 7-20. Recuperado de https://www.shorturl.at//ABHO

Zamudio Gómez, José. (2012). Epistemología y Educación. México: Red Tercer Milenio.

Zemelman, Hugo. (2011). Sujeto, existencia y potencia. Barcelona, España: Anthropos. 


\section{Revista indizada en}

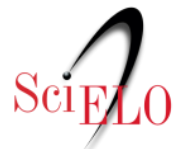

Distribuida en las bases de datos:

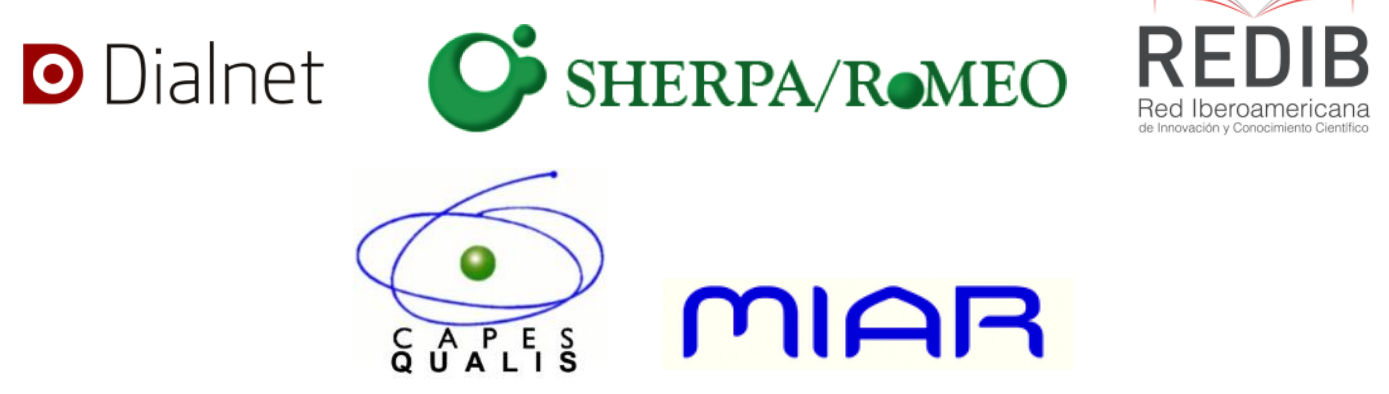

